\title{
Analysis of microRNA and gene networks in human chronic myelogenous leukemia
}

\author{
KUNHAO WANG ${ }^{1}$, ZHIWEN XU $^{2}$, NING WANG $^{2}$, YE TIAN $^{3}$, XIN SUN $^{4}$ and YONGGANG MA ${ }^{5}$ \\ ${ }^{1}$ School of Information Engineering, Changchun University of Science and Technology, Changchun, \\ Jilin 130600; ${ }^{2}$ College of Computer Science and Technology, Jilin University, Changchun, Jilin 130012; \\ ${ }^{3}$ School of Computer Science and Technology, Changchun University of Science and Technology, Changchun, \\ Jilin 130022; ${ }^{4}$ College of Information Science and Engineering, Ocean University of China, Qingdao, \\ Shandong 266100; ${ }^{5}$ College of Science, Dalian Ocean University, Dalian, Liaoning 116023, P.R. China
}

Received February 12, 2015; Accepted September 16, 2015

DOI: $10.3892 / \mathrm{mmr} .2015 .4502$

\begin{abstract}
Molecular biologists have identified a number of genes and microRNAs (miRs) associated with chronic myelogenous leukemia (CML). However, their underlying mechanisms in CML remain unclear. In the present study, three regulatory networks of genes and miRs were constructed to elucidate the underlying mechanisms of CML. The first network was the experimentally validated network of miRs and genes. The second was the dysregulatory network of CML, consisting of dysregulated genes and miRs, contributing to the pathogenesis of CML. The third was the CML-associated network, consisting of CML-associated genes and miRs. In addition to dysregulated genes and miRs, the associated network includes non-dysregulated genes and miRs that contribute to prevention, diagnosis, metastasis and therapy of CML. Key pathways were extracted and compared to distinguish the similarities and differences between dysregulatory nodes among the three networks. V-myb avian myeloblastosis viral oncogene homolog and miR-155 were observed to form a feedback loop module in the dysregulatory network. Regulation of the dysregulatory network may present as a strategy for gene therapy of CML. The current study provides an improved understanding of the molecular mechanisms of, and a potential treatment strategy for, CML.
\end{abstract}

Correspondence to: Professor Zhiwen $\mathrm{Xu}$, College of Computer Science and Technology, Jilin University, 2699 Qianjin Street, Changchun, Jilin 130012, P.R. China

E-mail: xuzw@jlu.edu.cn

Abbreviations: miR, microRNA; TFs, transcription factors; CML, chronic myelogenous leukemia; NCBI, National Center for Biotechnology Information; TFBSs, transcription factor binding sites; SNP, single nucleotide polymorphism

Key words: transcription factor, chronic myelogenous leukemia, microRNA, network, pathway, host gene

\section{Introduction}

Chronic myelogenous leukemia (CML) is the most common type of malignant cancer of the myeloproliferative neoplasms. CML accounts for $20 \%$ of all cases of leukemia in the global population (1). Numerous previous studies (2-4) demonstrated that genes and microRNAs (miRs) have various roles in CML. Dysregulated genes and miRs are important in the pathogenesis of CML, for example, the tumor protein 63 (TP63) mutation may function as a gene alteration and, thus, be responsible for the development of CML (5). Furthermore, downregulation of miR-10a may increase upstream stimulatory factor 2 expression levels and contribute to the increase in cell proliferation in CML (6). Genes and miRs that are associated with CML, but are non-dysregulated also have a role in CML, for example, runt-related transcription factor 3 (RUNX3) is involved in CML persistence despite imatinib treatment (7) and miR-30a may inhibit the effectiveness of imatinib-mediated apoptosis in CML (8).

Gene regulatory factors are predominantly transcription factors (TFs) and microRNAs (miRs), which control the expression of genomic information in multicellular genomes (9). TFs are proteins that bind to specific DNA sequences, controlling the encoding of genetic information from DNA to messenger RNA (10). TFs, alone or with other proteins, regulate gene expression by activation or suppression. miR is a small non-coding RNA molecule ( 22 nt in length), which functions in post-transcriptional regulation of gene expression and is also involved in transcriptional regulation (11). miRs regulate gene expression via silencing or target degradation and affect various cancer processes, including proliferation, differentiation and apoptosis.

miRs target thousands of genes, called target genes, and these are important for investigating the biological function of miRs. There are many methods to evaluate the regulatory associations between miRs and their target genes, such as microRNA.org (12), TargetScan (13) and PicTar (14). There are also experimentally validated databases, such as TarBase (15) miRTarBase (16) and miRecords (17), which provide abundant data to evaluate the associations between miRs and genes. 
miRs may be encoded in the DNA sequence of a gene, known as a host gene. During the transcription process, miRs and their host gene are transcribed simultaneously (18). The host gene and its intronic miR are often involved in the same biological processes (19). The two perform certain functions and are involved in signaling pathways (20).

The onset of CML may result from numerous dysregulated genes and miRs. Many genes (21) and miRs (22) associated with CML have been identified; however, the underlying mechanisms in CML remain unclear. The present study focused on the network of TFs, miRs, and their target and host genes, to establish the key pathways of CML and partly indicate their control mechanisms in CML. Experimentally validated regulatory associations (TFs $\rightarrow$ miRs, miRs $\rightarrow$ target genes, and host genes $\rightarrow$ miRs) were collected from known databases $(15-17,23)$. Dysregulated genes and miRs, as well as CML-associated genes and miRs were collected from known databases and the PubMed database (http://www. ncbi.nlm.nih.gov/pubmed/). To further investigate the transcription process of CML, TFs were selected by the P-Match method (24) and considered as CML-associated genes. Three networks were constructed to demonstrate the regulatory mechanism of CML. The first network is the network of miRs and genes from experimentally validated data. The second network is the dysregulatory network, which consists of dysregulatory data (genes and miRs) and their regulatory associations from the first network. The third is the CML-associated network, which consists of CML-associated data (genes and miRs) with their regulatory associations from the first network. In the current study, regulatory associations are referred to as pathways. To allow comparison of the similarities and differences distinguishing the key pathways in CML, the dysregulated gene and miR pathways, and predicted TFs were extracted from the three networks separately. These pathways of dysregulated genes and miRs may be particularly important in the development of CML (25), as the dysregulatory network contributes to the pathogenesis of CML (with regard to genes and miRs) while the associated network describes the regulatory mechanisms of CML.

\section{Materials and methods}

Material collection and data processing. An experimentally validated dataset of miRs and their target genes were collected from Tarbase version 5.0 (15), miRTarBase version 3.5 (16) and miRecords version 4 (17). The National Center for Biotechnology Information (NCBI) gene database (http://www.ncbi.nlm.nih.gov/gene/) was used to unify official symbols of miRs and genes. This dataset was designated as set $U_{1}$. An experimentally validated dataset between TFs and miRs was collected from TransmiR version 1.2 (23). This dataset was designated as set $\mathrm{U}_{2}$. A dataset of host genes and their miRs was collected from the NCBI gene database, and this dataset was designated as set $\mathrm{U}_{3}$.

In the present study, the dysregulated genes include genes with mutations, abnormal expression and single nucleotide polymorphisms, and inactivated, overexpressed, underexpressed, downregulated, upregulated and differentially expressed genes. The dataset of dysregulated genes was collected from Cancer Genetics Web (http://www.cancerindex.org/geneweb/index.html), the KEGG pathway database (26) and the PubMed database. CML-associated genes include dysregulated genes, and genes associated with prevention and radial therapy $(27,28)$. A dataset of CML-associated genes was collected from the GeneCards database (29), the PubMed database and included dysregulated genes. To improve understanding of the transcriptional network involving TFs, miRs and targets genes, predicted TFs were extracted using the P-Match method (24). These TFs are suggested as CML-associated genes. In the present study, TFs that appear in TransmiR were focused on. Promoter region sequences $(1,000 \mathrm{nt})$ of target genes that are targeted by dysregulated miRs were downloaded from University of California, Santa Cruz, Genome Browser (30). The P-Match method was used to identify TF binding sites (TFBSs) in the 1,000-nt promoter region sequences and the TFBSs were mapped onto the 1,000-nt promoter region of target genes; the corresponding TFs were obtained using these TFBSs. Matrix libraries of P-Match data, in addition to sets of known TF-binding sites collected in TRANSFAC ${ }^{\circledR}(31)$ enabled the identification of a large variety of TF binding sites. The vertebrate matrix was used with restricted high quality criterion for the matrix. The dataset of dysregulated genes was designated as set $\mathrm{U}_{4}$ and the associated genes were designated as set $\mathrm{U}_{5}$.

Dysregulated miRs include deletions, mutations, and differential, overexpressed, low expression, and down- and upregulated miRs. The dataset of dysregulated miRs was collected from the PubMed database. This dataset was designated as set $\mathrm{U}_{6}$. The associated miRs are involved in various CML processes and include dysregulated and non-dysregulated miRs. The dataset of associated miRs were collected from the PubMed database and the HMDD database 2.0 (32). This dataset was designated as set $\mathrm{U}_{7}$.

Construction of the three networks. Experimentally validated, dysregulatory and associated networks were constructed. Regulatory associations of TFs, miRs, target and host genes were extracted from sets $\mathrm{U}_{1}, \mathrm{U}_{2}$ and $\mathrm{U}_{3}$, these nodes were combined and their associations used to construct the experimentally validated network. Dysregulated genes and miRs were extracted from sets $\mathrm{U}_{4}$ and $\mathrm{U}_{6}$, and mapped onto the experimentally validated network. These genes, miRs and host genes, as well as their associations, were constructed into the dysregulatory network using the above data and other dysregulated data (genes and miRs), which do not have regulatory associations with other genes and miRs.

A similar method was used to construct the associated network. Associated genes and miRs were extracted from sets $\mathrm{U}_{5}$ and $\mathrm{U}_{6}$, and were mapped onto an experimentally validated network. These genes, miRs, host genes and their associations were extracted, and the associated network was constructed using the above data and other associated data (genes and miRs), which do not have regulatory associations with other genes and miRs.

\section{Results}

Dysregulatory network of CML. Dysregulated miRs and genes may result in the onset of CML. Fig. 1 presents the 


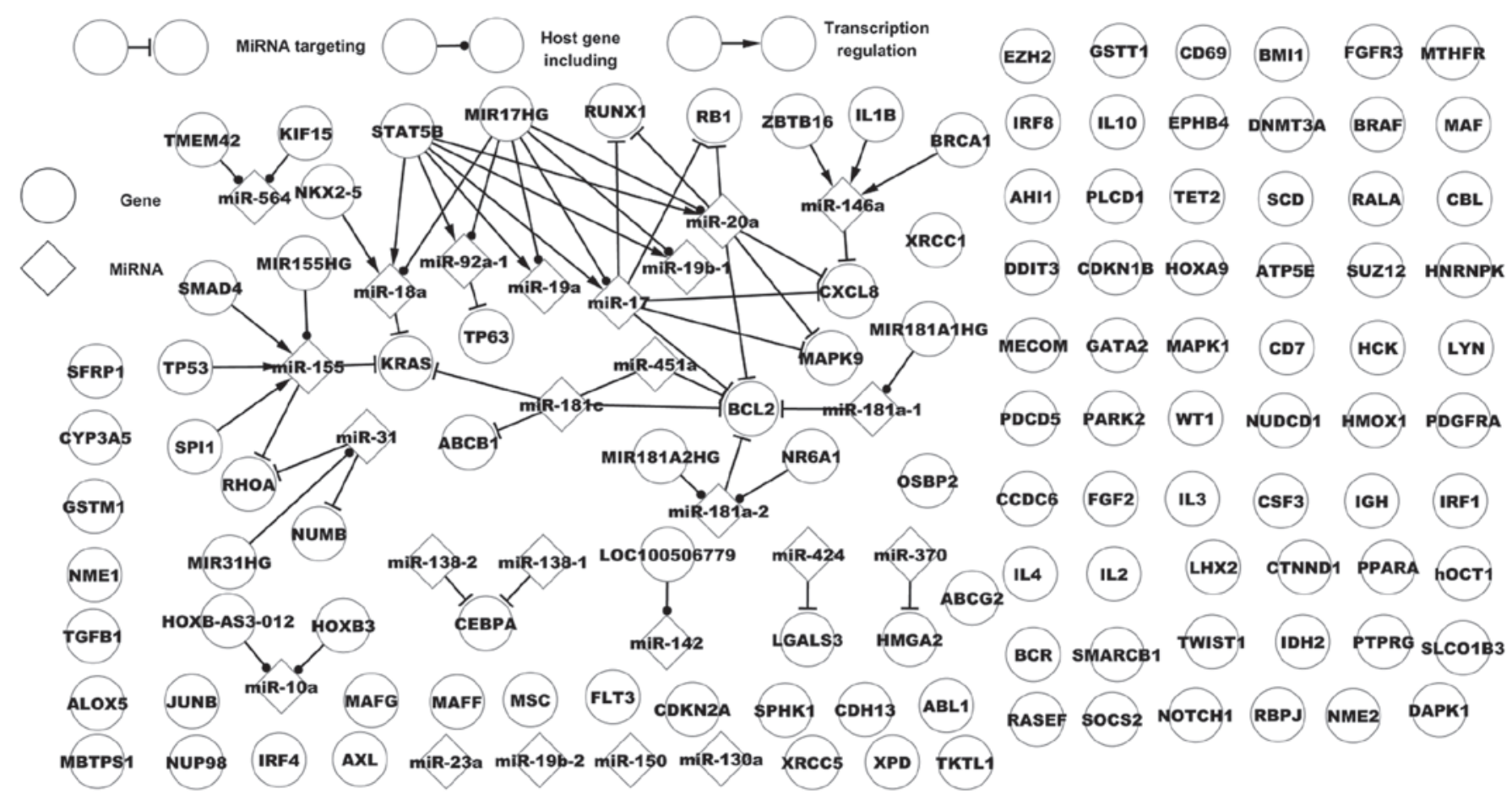

Figure 1. Dysregulatory network of chronic myelogenous leukemia. TMEM42, transmembrane protein 42; KIF15, kinesin family member 15; STAT5B, signal transducer and activator of transcription 5B; MIR17HG, miR-17-92 cluster host gene; RUNX1, runt-related transcription factor 1; RB1, retinoblastoma 1; NKX2-5, NK2 homeobox 5; IL8, interleukin-8; TP63, tumor protein 63; MIR181A1HG, MIR181A1 host gene; MAPK9, mitogen-activated protein kinase 9; $T P 53$, tumor protein 53; KRAS, Kirsten rat sarcoma viral oncogene homolog; BCL2, B-cell CLL/lymphoma 2; SPI1, Spi-1 proto-oncogene.

dysregulatory network of CML. This network includes 31 genes, $20 \mathrm{miRs}, 50$ of their regulatory associations, 89 single nodes (genes) and four single nodes (miRs). Fig. 1 presents various types of regulatory associations between miRs and genes: One miR may target one or numerous genes, one TF may regulate one or numerous miRs, several TFs may regulate one or numerous miRs and numerous miRs may target one or numerous genes. This dysregulatory network partly demonstrates the regulatory mechanism of CML.

Certain features of host genes and their miRs are presented in Fig. 1. A host gene includes one miR, which targets a number of genes; for example, MIR181A1 host gene (MIR181A1HG) includes miR-181a-1 that targets B-cell CLL/lymphoma 2 (BCL2). One host gene may include many miRs with targets. One miR may be located in various genes, for example, transmembrane protein 42 and kinesin family member 15 include miR-564. Fig. 1 presents a host gene that includes multiple miRs that alone or together target a number of genes. A notable host gene, MIR17HG, contains six miRs, miR-20a, miR-17, miR-92a-1, miR-18a, miR-19a and miR-19b-1. Of these, three, miR-20a, miR-17 and miR-92a-1, target six genes, chemoikine (C-X-C motif) ligand $8(C X C L 8)$, mitogen-activated protein kinase (MAPK) 9, TP63, retinoblastoma (RB) 1, RUNX1 and $B C L 2$. The six miRs are regulated by signal transducer and activator of transcription 5B (STAT5B). miR-19a, miR-18a and miR-19b-1 do not target any dysregulated genes. In the present study, 10 dysregulated genes are host genes, however, their miRs are not associated with dysregulation in CML, for example, RUNX1 includes miR-802 that is not associated with dysregulation in CML. Although certain host genes are not associated with dysregulation they may be involved in CML.

Associated network of CML. There are numerous genes and miRs in the CML-associated network, as presented in Fig. 2, which demonstrates a portion of the associated network in CML. Similarities and differences between the dysregulatory network and the associated network could not be clearly observed, thus a subnetwork was used to examine the two networks. As presented in Fig. 3, the larger nodes represent dysregulated data (genes and miRs), while the smaller nodes represent non-dysregulated data (genes and miRs). Fig. 3 demonstrates the associated network including additional TFs, miRs, target genes and their additional pathways, such as the TP53 $\rightarrow$ miR-29a $\rightarrow$ protein phosphatase, $\mathrm{Mg} 2+/ \mathrm{Mn} 2+$ dependent, 1D (PPM1D) pathway. TP53 is a dysregulated gene in CML, while miR-29a and PPMID are non-dysregulated. In another pathway, Spi-1 proto-oncogene $(S P I 1) \rightarrow$ miR-155 $\rightarrow$ Fli-1 proto-oncogene, ETS transcription factor (FLI1), SPII and miR-155 have dysregulatory expression, while FLII is a non-dysregulated gene in CML. The associated network extends the dysregulatory network and these novel pathways may contribute to tumor growth, migration, development, or prevention, diagnosis and other processes in CML.

Transcriptional network of predicted TFs. Analysis of 11 dysregulated miRs that are regulated by predicted TFs was conducted. Fig. 4 presents six predicted TFs that regulate nine 
Table I. Regulatory associations between miRs and $M Y B$.

Network

Dysregulatory

Associated

Validated
$M Y B$ targeting miRs

$M Y B$ regulated miRs

\author{
miR-150, mir-155, mir-424 \\ miR-150, mir-155, mir-424 \\ mir-107, miR-150, miR-155, miR-15a, \\ miR-16-1, miR-16-2, miR-34a, miR-34b, \\ miR-34c, miR-424
}

$\operatorname{miR}-155$

$\operatorname{miR}-155$

miR-15a, miR-155, miR-148a

miR, microRNA; MYB, v-myb avian myeloblastosis viral oncogene homolog.

Table II. Regulatory associations between miR-20a and genes.

\begin{tabular}{|c|c|c|}
\hline Network & miR-20a regulating genes & miR-20a targeted genes \\
\hline Dysregulatory & STAT5B & $B C L 2, R U N X 1, M A P K 9, R B 1$ \\
\hline Associated & $\begin{array}{l}C C N D 1, E 2 F 1, \\
E S R 1, M Y C, N K X 2-5, \\
S P I 1, S T A T 5 B\end{array}$ & $\begin{array}{l}\text { CCND1, BCL2, RUNX1, HIF 1A, } \\
\text { CDKN1A, E2F1, E2F3, } \\
\text { MYC, NRAS, MAPK9, PTEN, } \\
\text { RB1, TGFBR2, THBS1, VEGFA }\end{array}$ \\
\hline Validated & $\begin{array}{l}C C N D 1, E 2 F 1, M Y C, \\
M Y C N, N K X 2-5, T L X 1, \\
T L X 3, E S R 1, S T A T 5 B, S P I 1\end{array}$ & $\begin{array}{l}\text { CDKN1A, E2F1, MUC17, } \\
\text { E2F3, HIF } 1 A, M Y C, \\
\text { SMAD4, APP, MEF } 2 D, \\
\text { RB1, NRAS, PTEN, } \\
\text { MAPK9, RBL1, RBL2, } \\
\text { CCND1, BMPR2, BNIP2, } \\
\text { TGFBR2, WEE1, THBS1, } \\
\text { VEGFA, MAP3K12, BCL2, } \\
\text { RUNX1, CCND2 }\end{array}$ \\
\hline
\end{tabular}

miR, microRNA; STAT5B, signal transducer and activator of transcription 5B; E2F1, E2F transcription factor 1; NKX2-5, NK2 homeobox 5; $S P I 1$, Spi-1 proto-oncogene; $B C L 2$, B-cell CLL/lymphoma 2; RUNX1, runt-related transcription factor 1; MAPK9, mitogen-activated protein kinase $9 ; R B 1$, retinoblastoma 1 .

dysregulated miRs, which, in turn, target nine dysregulated target genes in CML. Interferon regulatory factor 1 (IRF1) (33), paired box 5 (PAX5) (2), RUNX1 (34) and nuclear factor of kappa light polypeptide gene enhancer in B-cells 1 (NFKB1) (35) have been experimentally validated in CML. The present study focuses on $N F K B 1$, which regulates four miRs, miR-21, miR-17, miR-146a and miR-155. NFKB1 regulates miR-17, which targets $C X C L 8, R U N X 1, B C L 2$, retinoblastoma $1(R B 1)$ and mitogen-activated kinase 9 (MAPK9). E2F transcription factor 1 and NK2 homeobox 5 (NKX2-5) co-regulate miR-18a, which targets Kirsten rat sarcoma viral oncogene homolog (KRAS). In addition, Fig. 4 demonstrates that a dysregulated miR may be regulated by various TFs, a target gene may be targeted by numerous dysregulated miRs and a TF may indirectly affect other genes via certain dysregulated miRs, such as miR-155, which is regulated by v-rel avian reticuloendotheliosis viral oncogene homolog A (RELA) encoding p65, NFKB1 and IRF1. Three miRs, miR-451a, miR-20a and miR-17 target BCL2. NKX2-5 regulates miR-17 and miR-20a, which target $R U N X 1$.

Regulatory pathways of dysregulated genes. To improve understanding of regulatory pathways, regulatory pathways of dysregulated genes and miRs, and predicted TFs were extracted and compared according to their predecessors (a node preceding the current one in a pathway) and successors (a node following the current one in a pathway).

V-myb avian myeloblastosis viral oncogene homolog $(M Y B)$ served as an example of a dysregulated gene. Table I indicates $M Y B$, its predecessors and successors in the three networks. Three miRs target $M Y B$, which regulates miR-155 in the dysregulatory network and the association network. Ten miRs target $M Y B$, which regulates three miRs in the experimentally validated network. miR-155 and $M Y B$ form a feedback loop (FBL) module in the dysregulatory network. The FBL module is a specific pathway, in which a TF regulates an miR that targets the TF.

Regulatory pathways of dysregulated miRs. The pathways of each dysregulated miR were extracted and compared using the same method as for the dysregulated genes. Table II presents miR-20a, and its predecessors and successors in the three networks. STAT5B regulates miR-20a that targets four genes in the dysregulatory network. Seven TFs regulate miR-20a that targets 15 genes in the associated network. There are 
Table III. Regulatory associations between miRs and NFKB1.

Network

Dysregulatory

Associated

Validated
NFKBl targeting miRs

miR-146a

miR-146a

let-7a-1, let-7a-2, let-7a-3, miR-146a, miR-146b, miR-15a

miR-9-1, miR-9-2, miR-9-3
NFKB1 regulated miRs

miR-146a, miR-155, miR-17, miR-21

miR-146a, miR-155, miR-17, miR-21

miR-16-1, miR-16-2, miR-199a-2, miR-21, miR-214, miR-224, miR-29a, miR-29b-1, miR-29b-2, miR-29c, miR-34a, miR-365, miR-448, miR-9-1, miR-9-2, miR-9-3

miR, microRNA; NFKB1, nuclear factor of kappa light polypeptide gene enhancer in B-cells 1.

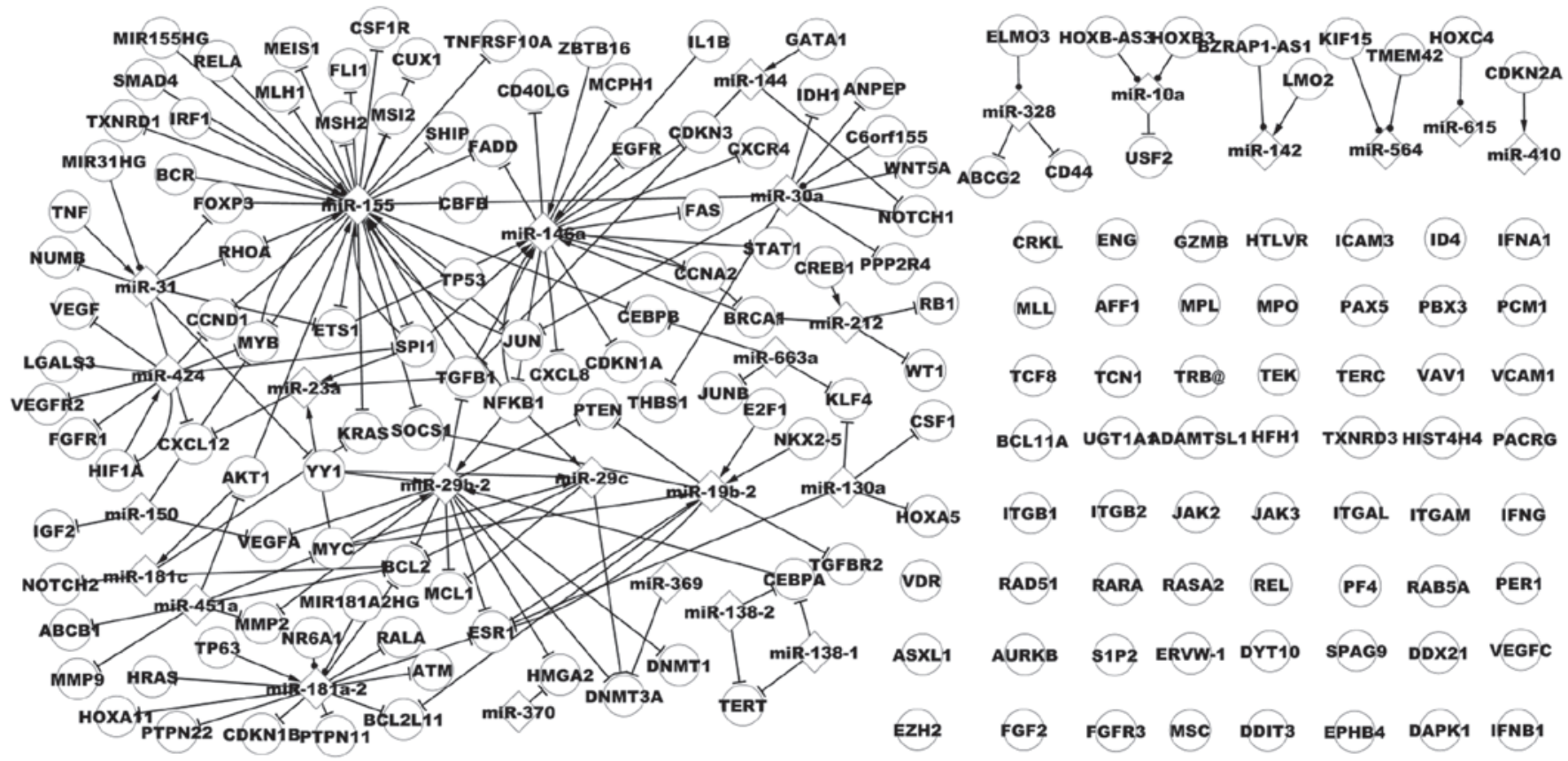

Figure 2. A subnet of associated network in chronic myelogenous leukemia. miR, microRNA; RELA, v-rel avian reticuloendotheliosis viral oncogene homolog A; FLI1, Fli-1 proto-oncogene, ETS transcription factor; IRF1, interferon regulatory factor 1; EGFR, epidermal growth factor receptor; TP53, tumor protein 53; $M Y B$, v-myb avian myeloblastosis viral oncogene homolog; SPI1, Spi-1 proto-oncogene; RB1, retinoblastoma 1; PAX5, paired box 5; IL8, interleukin-8; $N F K B 1$, nuclear factor of kappa light polypeptide gene enhancer in B-cells 1; E2F1, E2F transcription factor 1; KRAS, Kirsten rat sarcoma viral oncogene homolog; NKX2-5, NK2 homeobox 5; BCL2, B-cell CLL/lymphoma 2; TP63, tumor protein p63; BCL2L11, BCL2-like 11.

10 TFs, which regulate miR-20a that targets 26 genes in the experimentally validated network.

Regulatory pathways of predicted TFs. The same method was used to extract and compare the pathways of each predicted TF. E2F1 and NFKB1, as well as three dysregulated miRs, form three FBL modules. Notably, IRFI and RUNXI are dysregulated genes in CML whereas NFKBI and $P A X 5$ are associated with CML.

Table III presents $N F K B 1$, and its predecessors and successors. Dysregulated miR (miR-146a) targets NFKB1, which, in turn, regulates four dysregulated miRs. miR-146a targets $N F K B 1$, which, in turn, regulates four miRs in the associated network. Nine miRs target $N F K B 1$, which regulates 24 miRs in the experimentally validated network. miR-146a and NFKB1 form an FBL module; NFKB1 is an associated gene in CML and miR-146a is a dysregulated miR.

\section{Discussion}

In the present study, the regulatory network consisted of dysregulated genes and miRs, termed a CML dysregulated expression network. In this network, certain dysregulated genes and miRs are involved in the occurrence of CML $(5,6)$. Therefore, these dysregulated genes and miRs as well as their associations may contribute to the pathogenesis of CML.

In the current study, significant pathways of dysregulated genes and $\mathrm{miRs}$ were observed in CML, such as miR-17 $\rightarrow$ BCL2, $I R F 1 \rightarrow \mathrm{miR}-155$ and $\mathrm{miR}-150 \rightarrow M Y B \rightarrow \mathrm{miR}-155$. These pathways may exert key biological functions in CML and may be involved in the development of CML. Certain pathways have been observed to influence processes in CML, for example, miR-150 $\rightarrow$ MYB contributes to Bcr-Abl-mediated transformation in CML (25). Certain pathways have not been observed in CML, however, as they influence specific processes of other 

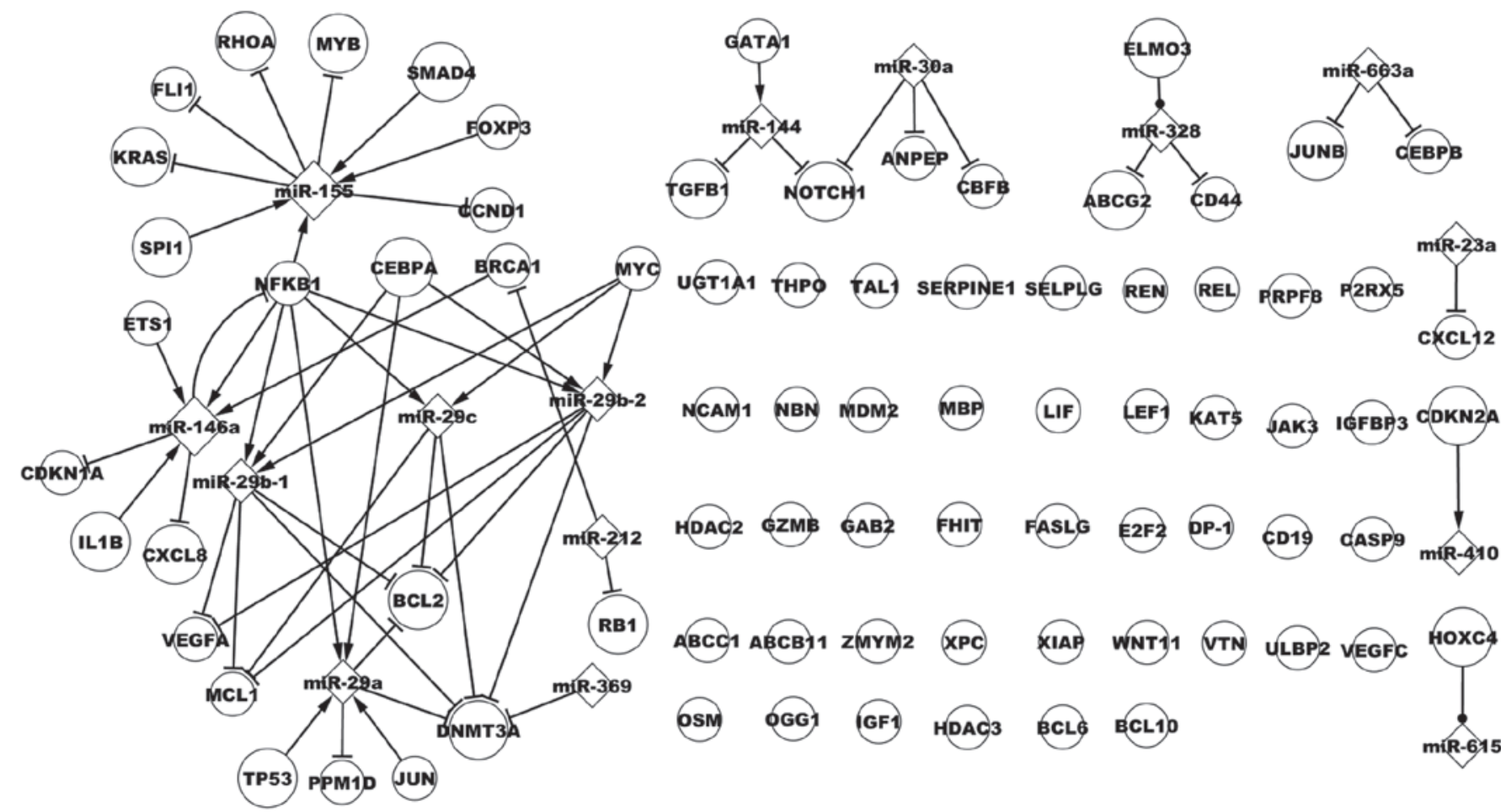

NCAM1 NBN MDMP MBP LIF LEF KATS

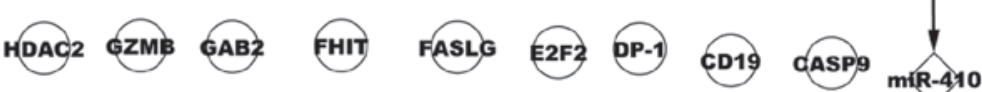
ABCG1 ABCBM1 Z OSM OGG GFI HDAG3 BCLO BCLID

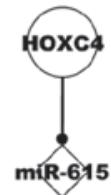

Figure 3. Similarities and differences between dysregulatory network and associated network in chronic myelogenous leukemia. miR, microRNA; $M Y B$, v-myb avian myeloblastosis viral oncogene homolog; FLII, Fli-1 proto-oncogene, ETS transcription factor; KRAS, Kirsten rat sarcoma viral oncogene homolog; SPII, Spi-1 proto-oncogene; $N F K B 1$, nuclear factor of kappa light polypeptide gene enhancer in B-cells 1; IL8, interleukin-8; BCL2, B-cell CLL/lymphoma 2; RB1, retinoblastoma 1; TP53, tumor protein 53; PPM1D, protein phosphatase, $\mathrm{Mg} 2+/ \mathrm{Mn} 2+$ dependent, $1 \mathrm{D}$.

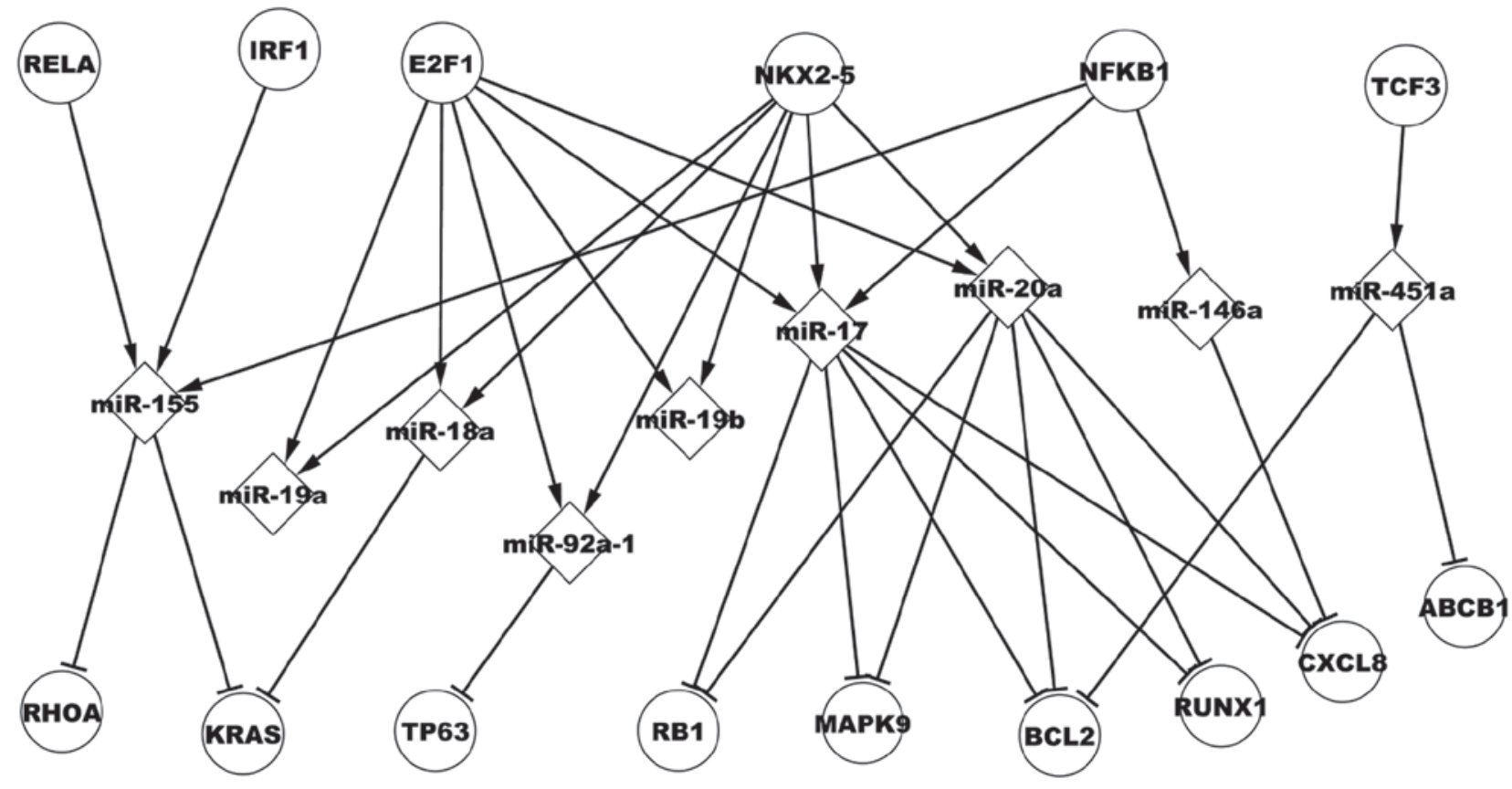

Figure 4. Regulatory associations of predicted transcriptional network in chronic myelogenous leukemia. RELA, v-rel avian reticuloendotheliosis viral oncogene homolog A; IRF1, interferon regulatory factor 1; E2F1, E2F transcription factor 1; NKX2-5, NK2 homeobox 5; KRAS, Kirsten rat sarcoma viral oncogene homolog; TP63, tumor protein 63; RB1, retinoblastoma 1; MAPK9, mitogen-activated protein kinase 9; $B C L 2$, B-cell CLL/lymphoma 2; RUNX1, runt-related transcription factor $1 ; I L 8$, interleukin-8.

types of cancer, their biological functions may contribute to CML. For example, miR-19a $\rightarrow$ BCL2-like 11 in T cell acute lymphoblastic leukemia (36) or epidermal growth factor receptor $\rightarrow$ miR-21 in age- and mutagen-associated changes in colon cancer stem-like cells (37). The remaining pathways that have not been identified in cancers may function in CML, 
for example $I R F 1 \rightarrow$ miR-155. For the pathways of predicted TFs, certain pathways have been determined in other types of carcinoma, for example $N K X 2-5 \rightarrow$ miR-17-92 where the TFs concomitantly reduce $E 2 F 1$, thereby enhancing survival of leukemic T cells (38).

In the dysregulated network, genes and miRs exhibit dysregulated expression. If this dysregulated expression pattern was to revert to a normal expression pattern, CML may not occur. Regulation of the dysregulated network may be an efficient gene therapy strategy for the treatment of CML. Future studies will focus on the signaling pathways composed of dysregulated genes and miRs, and explore how these pathways function in CML.

\section{Acknowledgements}

The present study was supported by the Jilin Sino-Rok Institute of Animal Science, Changchun Science and Technology (grant no. 14KG066), and the Jilin Provincial Science and Technology Department (grant no. 20130302012NY).

\section{References}

1. Vardiman JW: The World Health Organization (WHO) classification of tumors of the hematopoietic and lymphoid tissues: An overview with emphasis on the myeloid neoplasms. Chem Biol Interact 184: 16-20, 2010.

2. Kęsy $J$ and Januszkiewicz-Lewandowska D: Genes and childhood leukemia. Postepy Hig Med Dosw (Online) 69: 302-308, 2015.

3. Gao S, Bajrami I, Verrill C, Kigozi A, Ouaret D, Aleksic T, Asher R, Han C, Allen P, Bailey D, et al: Dsh homolog DVL3 mediates resistance to IGFIR inhibition by regulating IGF-RAS signaling. Cancer Res 74: 5866-5877, 2014.

4. Rokah OH, Granot G, Ovcharenko A, Modai S, Pasmanik-Chor M, Toren A, Shomron N and Shpilberg O: Downregulation of miR-31, miR-155, and miR-564 in chronic myeloid leukemia cells. PLoS One 7: e35501, 2012.

5. Yamaguchi H, Inokuchi K, Sakuma Y and Dan K: Mutation of the p51/p63 gene is associated with blastic crisis in chronic myelogenous leukemia. Leukemia 15: 1729-1734, 2001.

6. Agirre X, Jiménez-Velasco A, San José-Enériz E, Garate L, Bandrés E, Cordeu L, Aparicio O, Saez B, Navarro G, Vilas-Zornoza A, et al: Down-regulation of hsa-miR-10a in chronic myeloid leukemia CD34+cells increases USF2-mediated cell growth. Mol Cancer Res 6: 1830-1840, 2008.

7. Miething C, Grundler R, Mugler C, Brero S, Hoepfl J, Geigl J, Speicher MR, Ottmann O, Peschel C and Duyster J: Retroviral insertional mutagenesis identifies RUNX genes involved in chronic myeloid leukemia disease persistence under imatinib treatment. Proc Natl Acad Sci USA 104: 4594-4599, 2007.

8. Yu Y, Yang L, Zhao M, Zhu S, Kang R, Vernon P, Tang D and Cao L: Targeting microRNA-30a-mediated autophagy enhances imatinib activity against human chronic myeloid leukemia cells. Leukemia 26: 1752-1760, 2012.

9. Hobert O: Gene regulation by transcription factors and microRNAs. Science 319: 1785-1786, 2008.

10. Latchman DS: Transcription factors: An overview. Int J Biochem Cell Biol 29: 1305-1312, 1997.

11. Chen K and Rajewsky N: The evolution of gene regulation by transcription factors and microRNAs. Nat Rev Genet 8: 93-103, 2007.

12. Betel D, Wilson M, Gabow A, Marks DS and Sander C: The microRNA.org resource: Targets and expression. Nucleic Acids Res 36 (Database): D149-D153, 2008.

13. Lewis BP, Burge CB and Bartel DP: Conserved seed pairing, often flanked by adenosines, indicates that thousands of human genes are microRNA targets. Cell 120: 15-20, 2005

14. Krek A, Grün D, Poy MN, Wolf R, Rosenberg L, Epstein EJ, MacMenamin P, da Piedade I, Gunsalus KC, Stoffel M and Rajewsky J: Combinatorial microRNA target predictions. Nat Genet 37: 495-500, 2005.
15. Papadopoulos GL, Reczko M, Simossis VA, Sethupathy P and Hatzigeorgiou AG: The database of experimentally supported targets: A functional update of TarBase. Nucleic Acids Res 37 (Database): D155-D158, 2009.

16. Hsu SD, Lin FM, Wu WY, Liang C, Huang WC, Chan WL, Tsai WT, Chen GZ, Lee CJ, Chiu CM, et al: miRTarBase: A database curates experimentally validated microRNA-target interactions. Nucleic Acids Res 39 (Database): D163-D169, 2011.

17. Xiao F, Zuo Z, Cai G, Kang S, Gao X and Li T: miRecords: An integrated resource for microRNA-target interactions. Nucleic Acids Res 37 (Database): D105-D110, 2009.

18. Rodriguez A, Griffiths-Jones S, Ashurst JL and Bradley A: Identification of mammalian microRNA host genes and transcription units. Genome Res 14 (10A): 1902-1910, 2004.

19. Baskerville S and Bartel DP: Microarray profiling of microRNAs reveals frequent coexpression with neighboring miRs and host genes. RNA 11: 241-247, 2005.

20. Cao G, Huang B, Liu Z, Zhang J, Xu H, Xia W, Li J, Li S, Chen L, Ding H, et al: Intronic miR-301 feedback regulates its host gene, ska2, in A549 cells by targeting MEOX2 to affect ERK/CREB pathways. Biochem Biophy Res Commun 396: 978-982, 2010

21. Vaidya S, Ghosh K, Shanmukhaiah C, Vundinti BR: Genetic variations of hOCT1 gene and CYP3A4/A5 genes and their association with imatinib response in Chronic Myeloid Leukemia.Eur J Pharmacol 765: 124-130, 2015.

22. Fallah P, Amirizadeh N, Poopak B, Toogeh G, Arefian E, Kohram F, Hosseini Rad SM, Kohram M, Teimori Naghadeh H and Soleimani M: Expression pattern of key microRNAs in patients with newly diagnosed chronic myeloid leukemia in chronic phase. Int J Lab Hematol 37: 560-568, 2015.

23. Wang J, Lu M, Qiu C and Cui Q: TransmiR: A transcription factor-microRNA regulation database. Nucleic Acids Res 38 (Database): D119-D122, 2010.

24. Chekmenev DS, Haid C and Kel AE: P-Match: transcription factor binding site search by combining patterns and weight matrices. Nucleic Acids Res 33 (Web Server): W432-W437, 2005.

25. Flamant S, Ritchie W, Guilhot J, Holst J, Bonnet ML, Chomel JC, Guilhot F, Turhan AG and Rasko JE: Micro-RNA response to imatinib mesylate in patients with chronic myeloid leukemia. Haematologica 95: 1325-1333, 2010.

26. Kanehisa M and Goto S: KEGG: Kyoto encyclopedia of genes and genomes. Nucleic Acids Res 28: 27-30, 2000.

27. Dong W, Zhang J, Shao N, Tian T, Li L, Jian J, Zang S, Ma D and Ji C: Development and immunological evaluation of HLA-specific chronic myeloid leukemia polyepitope vaccine in Chinese population. Vaccine 32: 3501-3508, 2014.

28. Sekiguchi Y, Shimada A, Matsuzawa M, Imai H, Wakabayashi M, Sugimoto K, Nakamura N, Sawada T, Arita J, Komatsu N and Noguchi M: Occurrence of carcinoma of the pancreas following nilotinib therapy for chronic myeloid leukemia: Report of a case with review of the literature. Turk $\mathrm{J}$ Haematol 32: 257-262, 2015.

29. Safran M, Dalah I, Alexander J, Rosen N, Iny Stein T, Shmoish M, Nativ N, Bahir I, Doniger T and Krug H: GeneCards version 3: The human gene integrator. Database (Oxford) baq020, 2010.

30. Fujita PA, Rhead B, Zweig AS, Hinrichs AS, Karolchik D, Cline MS, Goldman M, Barber GP, Clawson $\mathrm{H}$ and Coelho A: The UCSC Genome Browser database: Update 2011. Nucleic Acids Res 39 (Database): D876-D882, 2011.

31. Matys V, Fricke E, Geffers R, Gössling E, Haubrock M, Hehl R, Hornischer K, Karas D, Kel AE, Kel-Margoulis OV, et al: TRANSFAC: Transcriptional regulation, from patterns to profiles. Nucleic Acids Res 31: 374-378, 2003.

32. Li Y, Qiu C, Tu J, Geng B, Yang J, Jiang T and Cui Q: HMDD v2.0: A database for experimentally supported human microRNA and disease associations. Nucleic Acids Res 42 (D1): D1070-D1074, 2014.

33. Tzoanopoulos D, Speletas M, Arvanitidis K, Veiopoulou C, Kyriaki S, Thyphronitis G, Sideras P, Kartalis G and Ritis K: Low expression of interferon regulatory factor-1 and identification of novel exons skipping in patients with chronic myeloid leukaemia. Br J Haematol 119: 46-53, 2002.

34. Zhao LJ, Wang YY, Li G, Ma LY, Xiong SM, Weng XQ, Zhang WN, Wu B, Chen Z and Chen SJ: Functional features of RUNX1 mutants in acute transformation of chronic myeloid leukemia and their contribution to inducing murine full-blown leukemia. Blood 119: 2873-2882, 2012. 
35. Reuter S, Charlet J, Juncker T, Teiten MH, Dicato M, Diederich M: Effect of curcumin on nuclear factor kappaB signaling pathways in human chronic myelogenous K562 leukemia cells. Ann N Y Acad Sci 1171: 436-447, 2009.

36. Ye H, Liu X, Lv M, Wu Y, Kuang S, Gong J, Yuan P, Zhong $\mathrm{Z}$, Li Q, Jia $\mathrm{H}$, et al: MicroRNA and transcription factor co-regulatory network analysis reveals miR-19 inhibits CYLD in T-cell acute lymphoblastic leukemia. Nucleic Acids Res 40: 5201-5214, 2012.
37. Nautiyal J, Du J, Yu Y, Kanwar SS, Levi E and Majumdar AP: EGFR regulation of colon cancer stem-like cells during aging and in response to the colonic carcinogen dimethylhydrazine. Am J Physiol Gastrointest Liver Physiol 302: G655-G663, 2012. 38. Nagel S, Venturini L, Przybylski GK, Grabarczyk P, Schmidt CA, Meyer C, Drexler HG, Macleod RA and Scherr M: Activation of miR-17-92 by NK-like homeodomain proteins suppresses apoptosis via reduction of E2F1 in T-cell acute lymphoblastic leukemia. Leuk Lymphoma 50: 101-108, 2009. 